\title{
Differential Susceptibility of Naïve and Activated Human $\gamma \delta$ T Cells to Activation-Induced Cell Death by T-Cell Receptor Cross-Linking
}

\author{
Yunn-Hwen Gan ${ }^{1}$, Shirley S.N. Lui ${ }^{1}$ and Miroslav Malkovsky ${ }^{2}$ \\ ${ }^{1}$ Department of Biochemistry, National University of Singapore \\ ${ }^{2}$ Department of Medical Microbiology and Immunology, University of Wisconsin-Madison, USA \\ Accepted August 10, 2001
}

\begin{abstract}
Background: $\mathrm{T}$ cells undergo activation-induced cell death (AICD) through repeated stimulation of their $\mathrm{T}$ cell receptors (TCRs). Activated human $\gamma \delta$ T cells were found to die by apoptosis when their TCRs were cross-linked by antibodies, whereas naïve $\gamma \delta \mathrm{T}$ cells freshly isolated from blood did not. Therefore, we investigated the factors that could contribute to this differential susceptibility.

Materials and Methods: $\gamma \delta$ T cells were isolated from the peripheral blood of healthy human volunteers and their TCRs were cross-linked either directly (naïve) or after an in vitro incubation of 11 days (activated). Their cell cycle profiles, cytokine, Fas and FasL mRNA messages, and surface expression of Fas and FasL were determined.

Results: The naïve cells were cycling while the activated $T$ cells exited from the Gl to subGl phase upon TCR
\end{abstract}

cross-linking. IL-2 and IL-4 mRNAs and surface expression of FasL were detected only in activated $T$ cells in the time period examined. In addition, cFLIP mRNA expression was found only in naïve $\gamma \delta \mathrm{T}$ cells and activated $\mathrm{T}$ cells treated with cyclosporin A (CsA), which inhibited AICD in the activated T cells. CsA also downregulated the surface expression of FasL in activated T cells.

Conclusions: The differential expression of cytokines, apoptotic inducers and inhibitors provide the basis for the differential susceptibility of naïve and activated $\gamma \delta$ T cells to AICD upon TCR cross-linking. This contributes to our understanding of the regulation and maintenance of $\gamma \delta$ T-cell homeostasis, which would be important in many infectious as well as autoimmune diseases, where $\gamma \delta$ $\mathrm{T}$ cells have been implicated.

\section{Introduction}

Activation-induced cell death (AICD) of lymphocytes is an important mechanism for homeostatic regulation of lymphocyte activation and proliferation (1) and for maintaining peripheral tolerance to self-antigens (2). AICD of T cells describes a process whereby repeated stimulation through the $\mathrm{T}$ cell receptor (TCR) results in apoptotic death mediated through Fas (CD95) and FasL (CD95L) interactions (3-5). It is a tightly regulated process and involves both apoptosis-inducing and inhibiting moieties, accounting for the AICD-susceptible or resistant phenotypes seen in different $T$ cells $(6,7)$. For example, naïve $T$ cells stimulated through their TCR do not undergo AICD due to high levels of cellular FLICE-like inhibitor protein (CFLIP), which competitively inhibits the binding of FLICE (FADD-like IL- $1 \beta$-converting enzyme), a zymogen of caspase 8 , and shuts off Fas signaling (8). It is also known that IL-2 regulates and potentiates AICD (9), and naïve $T$ cells need to progress into $S$ phase of the cell cycle before becoming susceptible (10).

Address correspondence and reprint requests to Dr. M. Malkovsky, University of Wisconsin Medical School, Department of Medical Microbiology and Immunology, 1300 University Avenue, Madison, WI 53706, USA. Phone \& fax: 608-263-6316; e-mail: mmalkovs@facstaff.wisc.edu
However, most studies examining AICD were done using $\mathrm{CD} 4^{+} \alpha \beta \mathrm{T}$ cells or T cell hybridomas. Little is known about the regulation of AICD in $\gamma \delta$ $\mathrm{T}$ cells, although AICD has been reported in murine intestinal intraepithelial $\gamma \delta \mathrm{T}$ cells and was shown to be augmented by IL-2 (11). $\gamma \delta \mathrm{T}$ cells isolated from Mycobacteria tuberculosis-infected patients were also more susceptible to AICD than normal individuals and death was mediated by Fas and FasL interactions (12).

$\gamma \delta \mathrm{T}$ cells are involved in natural anti-infectious immunity $(13,14)$. They rapidly respond in an MHCunrestricted manner to phosphate-containing nonpeptide molecules (15-17) and alkylamines (18) present in many microbes. Therefore, in addition to the control through T-cell co-receptors (19), the regulation of AICD in these cells may be an important element in guiding innate T-cell immunity responses. We have compared the cell cycle and cytokine profiles, as well as the Fas, FasL and cFLIP expression of naïve versus activated $\gamma \delta \mathrm{T}$ cells stimulated by TCR cross-linking. This allows us to identify factors that could account for the differential susceptibility of naïve and activated $\gamma \delta$ T cells to AICD.

\section{Materials and Methods Cell Culture}

Human peripheral blood lymphocytes (PBL) were isolated from the blood of healthy volunteers drawn 
by venipuncture on a Ficoll density gradient (Sigma, St. Louis, MO). The PBLs were cultured in RPMI 1640 medium supplemented with 10\% FBS (Biological Industries, Israel), $100 \mu \mathrm{g} / \mathrm{ml}$ penicillin, $100 \mathrm{U} / \mathrm{ml}$ streptomycin, $2 \mathrm{mM}$ L-glutamine (Sigma) and $50 \mathrm{U} / \mathrm{ml}$ of IL-2 (GibcoBRL, Gaithersburg, MD). To activate the $\gamma \delta \mathrm{T}$ cells, mitomycin C-treated Daudi cells were added to the culture at a ratio of 1 Daudi cell to 5 PBLs. On the sixth day of culture, half of the media was removed and replaced with an equal amount of new media.

\section{Stimulation and Magnetic Cell Sorting (MACS) of $\gamma \delta T$ Cells}

Stimulation of both naïve and activated $\gamma \delta \mathrm{T}$ cells by TCR cross-linking was accomplished through the MACS procedure. For activated cells that had been in culture for 11-12 days, the $\gamma \delta$ T cells were positively selected by MACS as described (20). Briefly, the cells were first stained with anti-TCR $\delta 1$ antibody from the supernatant of 5A6.E9 hybridoma (ATCC, Rockville, MD) followed by goat-anti-mouse $\kappa$ antibody conjugated to biotin (PharMingen, San Diego, CA) and streptavidin microbeads (Miltenyi biotec, Sunnyvale, CA). To stimulate and purify naïve $\gamma \delta$ T cells, PBLs isolated from blood were subjected to MACS as above without prior in vitro culture. The eluted positive fraction typically contained $\gamma \delta$ T cells with a purity greater than $\mathbf{9 0 \%}$. The cells were plated in media and aliquots were taken out for subsequent experiments at various time-points after the separation. For cyclosporin A (CsA) (Sigma) treatment, cells were preincubated in CsA for 1 hour before stimulation and CsA was added to the purified cells plated in wells after MACS.

\section{Apoptosis of $\gamma \delta T$ Cells}

The percentage of apoptotic cells at each time point was determined by double staining of cells with annexin V-FITC and PI as described in the ApoAlert Annexin V FITC detection kit (Clontech, Palo Alto, CA). The bright staining PI cells were excluded as necrotic cells. Cells were analyzed by flow cytometry (FACScan, Becton Dickinson, San Jose, CA). Apoptosis was confirmed by microscopy and the viability of cells was determined by trypan blue dye exclusion method.

\section{Cell Cycle and Flow Cytometric Analysis}

The cells $\left(0.8 \times 10^{6}\right)$ were fixed in $1 \%$ formaldehyde and permeabilised with a solution of $12.5 \%$ RPMI, $12.5 \%$ FBS and $52.5 \%$ ethanol overnight. To detect the DNA content of the cells, $10 \mu \mathrm{g} / \mathrm{ml}$ propidium iodide (PI) (Sigma) and $100 \mathrm{U} / \mathrm{ml}$ RNaseA (Sigma) were added for 1 hour at room temperature. To stain the surface of cells for Fas and FasL expression, cells were stained with anti-Fas ZB4 antibody (I munotech, Marseille, France) followed by rat-antimouse IgGl conjugated to PE (Becton Dickinson), and biotinylated anti-FasL NOK-1 antibody (PharMingen) followed by streptavidin-PE (Becton Dickinson). Cells were preincubated in $5 \mathrm{mM}$ EDTA for at least 4 hours before staining for FasL. The cell cycle positions and surface expression were assessed by flow cytometry.

\section{RT-PCR Analysis}

RNA was extracted from $1.2 \times 10^{6}$ cells at each timepoint using TRIzol reagent (GibcoBRL) according to manufacturer's instructions. The RNA was reverse transcribed into cDNA by incubating $5 \mu \mathrm{g}$ of RNA, $0.2 \mu \mathrm{M}$ oligo-dT, $1 \mu \mathrm{M}$ dNTPs, $12 \mathrm{U}$ RNAsin (Promega, Madison, WI) and $20 \mathrm{U}$ of Moloney murine leukemia virus reverse transcriptase (New England Biolabs, Beverly, MA) at $37^{\circ} \mathrm{C}$ for 1 hour. PCR was performed using an $\mathrm{MJ}$-Research thermal cycler in a reaction mix consisting of cDNA, $0.4 \mu \mathrm{M}$ sense and anti-sense primers, $0.2 \mu \mathrm{M}$ dNTPs, $2 \mu \mathrm{M} \mathrm{MgCl}_{2}, 10 \mathrm{x}$ reaction buffer and 1 U Taq DNA polymerase (Biotools, Madrid, Spain). Each PCR amplification was carried out for 35 cycles. The primer sequences were as follows:

$\beta$-actin, $5^{\prime}$-AAATCGTGCGTGACATTAACG-3' and $5^{\prime}$-AGCACTGTGTTGGCGTACAG-3'; IL-2, 5'CATTGCACTAAGTCTTGCACTTGTCA-3' and ' $5^{\prime}$ CGTTGATATTGCTGATTAAGTCCCTG-3'; IL-4, 5' AACACAACTGAGAAGGAAACCTTC- $3^{\prime}$ and $5^{\prime}$-GCTCGAACACTTTGAATATTTCTC-3'; IL-7, 5' -ATGTTCCATGTTTCTTTTAGGTAT- ${ }^{\prime}$ and $5^{\prime}$-TGCATTTCTCAAATGCCCTAATCC-3'; IL-15, 5' -TGGAGCACAGAAATCAATG-3' and $5^{\prime}$-GAAACAGCCCAAAATGAAG-3'; TNF- $\alpha$, 5'-CAAGCCTGTAGCCCATGTTGTAGCA- ${ }^{\prime}$ and $5^{\prime}$-ATCCCAAAGTAGACCTGCCCAGACT-3'; IFN- $\gamma$, 5'-TTTGGGTTCTCTTGGCTGTTACTGC- $3^{\prime}$ and $5^{\prime}$-TTCGCTTCCCTGTTTTAGCTGCTGG-3'; Fas, 5'-ATCTGGACCCTCCTACCTCT-3' and 5'-AGATCTGGATCCTTCCTCTT-3'; FasL， 5'-GGATTGGGCCTGGGGATGTTTCA-3' and 5'-TTGTGGCTCAGGGGCAGGTTGTTG-3'; and cFLIP, 5'-GGGAGAAGTAAAGAACAAAG-3' and 5'-CGTAGGCACAATCACAGCAT- ${ }^{\prime}$.

The primers were all designed to give a different product length or none with genomic DNA. PCR products were size fractionated on $1 \%$ agarose gel and normalized according to the amount of $\beta$-actin detected in the same mRNA sample. The amount of cDNA template used for the reactions was predetermined by dilution to fall within the linear portion of amplification curve. Relative mRNA levels were determined using the BioProfile software of a photoimager (Vilber Lourmat, Marne La Vallee, France).

\section{Results}

Differential Susceptibility of Naïve and Activated $\gamma \delta$ T Cells to AICD

$\gamma \delta$ T cells that had been activated in vitro with IL-2 and Daudi cells (a Burkitt's lymphoma EBV-transformed cell-line known to be stimulatory for human $\gamma \delta$ $\mathrm{T}$ cells) underwent AICD when they were restimulated by TCR cross-linking during the positive MACS selection procedure (21). However, it was noticed that 


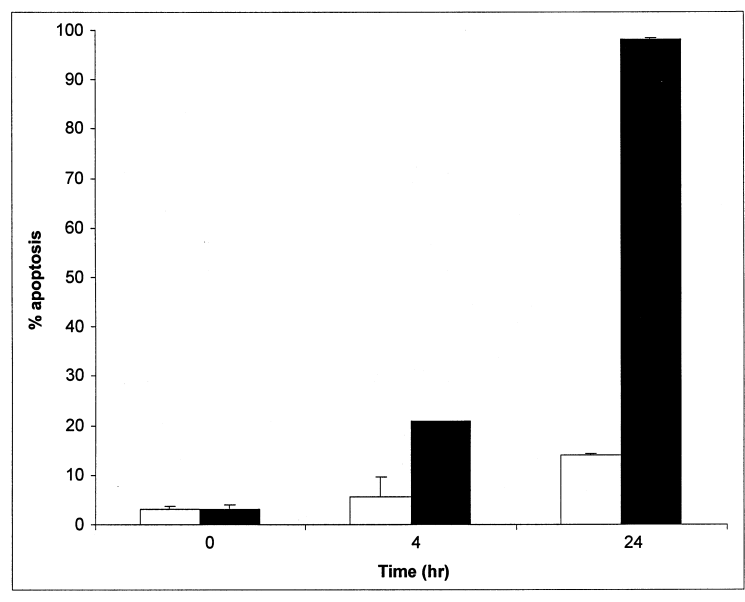

Fig. 1. Percentage of naïve and activated $\gamma \delta \mathrm{T}$ cells undergoing apoptosis at various time-points after TCR cross-linking. Results represent the average of duplicates at each timepoint and are representative of at least 3 different experiments. Percentage of apoptotic naïve $\gamma \delta \mathrm{T}$ cells (white bars $\pm \mathrm{SE}$ ) typically does not exceed $20 \%$ while apoptotic activated cells (black bars \pm SE) range from $50-98 \%$ in these experiments.

naïve $\gamma \delta \mathrm{T}$ cells isolated from blood and subjected to the same separation procedure without prior in vitro culture survived. The percentage of viability for naïve cells was typically around $70-90 \%$ while it ranged from $10-40 \%$ for activated cells at 24 hours after stimulation. The cells were found to die by apoptosis as determined by annexin V staining (Fig. 1). The percentage of apoptotic cells was below $20 \%$ for naïve cells while it ranged from $50-90 \%$ for activated $\gamma \delta$ $\mathrm{T}$ cells 24 hours after stimulation in different experiments. The same phenomenon was observed when naïve or activated cells were cross-linked with the same TCR antibodies, but without passing through the separation column. However, any TCR crosslinking mentioned in this study refers to the $\gamma \delta$ TCRs being cross-linked during the MACS procedure.

\section{Cell Cycle Profiles of $\gamma \delta T$ Cells after TCR Cross-Linking}

The cell cycle profiles of both naïve and activated $\gamma \delta$ $\mathrm{T}$ cells show that most cells remained in Gl phase of the cell cycle. In the naïve cell population, a portion of the cells entered into Gl 4 hours after stimulation and by 24 hours, some cells began to enter the $S$ phase (Fig. 2a), reflecting the asynchronous nature of the culture. In contrast, the activated cells showed a decline of cells in the Gl phase and a corresponding increase in cells exhibiting sub Gl DNA content (Fig. 2b). Very few cells were in the $S$ and G2/M phase throughout the time-points examined. The activated cells were exiting from Gl to the sub Gl phase. The percentage of apoptotic cells as assessed by cell cycle was always lower than that by annexin staining, this could be that the latter method detects cells early in apoptosis, while PI staining of DNA detects cells in late apoptosis and is less sensitive, as incomplete DNA loss resulted in a broader Gl peak (data not shown).
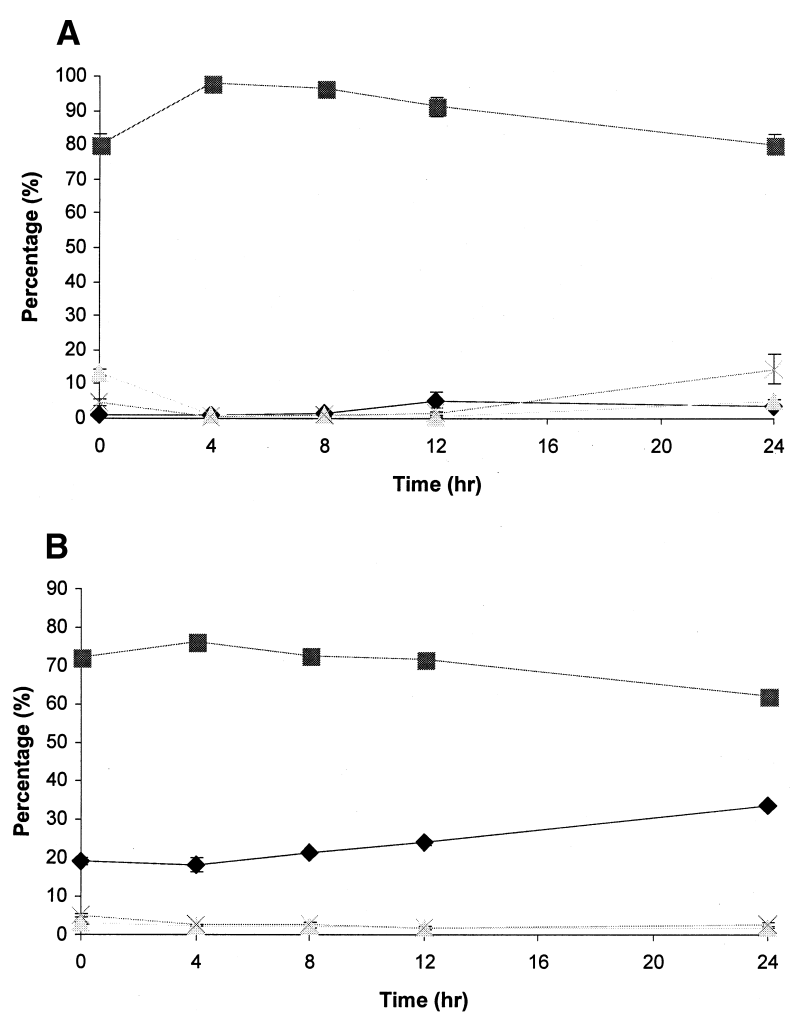

Fig. 2. Percentages of naïve and activated $\gamma \delta \mathrm{T}$ cells during various stages of cell cycle at different time-points after TCR cross-linking (A-naïve, B-activated). Each $\boldsymbol{\square} \times \triangle$ timepoint represents the average of duplicates of cells at Gl( ), S ( ), $\operatorname{G2} / \mathrm{M}()$ and sub Gl( ) phases of the cell cycle. The graphs are representative of at least 3 separate experiments.

\section{Differential mRNA Expression in Naïve} and Activated $\gamma \delta T$ Cells

Cytokines, most notably IL-2, have been implicated in driving cells into the cell cycle and therefore inducing AICD (9). We found that there were significant differences in the expression of cytokine messages between naïve and activated cells after TCR cross-linking (Table 1). The presence of IL-2 and IL-4 mRNAs 4 hours after cross-linking in activated $\gamma \delta \mathrm{T}$ cells was detected whereas they were absent in naïve T cells. IFN- $\gamma$ was detected only in activated $\mathrm{T}$ cells while TNF- $\alpha$ was detected in both activated and naïve T cells. Interestingly, TNF- $\alpha$ mRNA was well expressed in naïve $T$ cells and showed a 4-fold increase during the 24 hour observation period. In contrast, TNF- $\alpha$ mRNA was only detected after a 4-h stimulation in activated T cells (data not shown). In addition to IL-2 and IL-4, IL-7 and IL-15 can also support the growth and development of $\gamma \delta \mathrm{T}$ cells (22). However, they could not be detected in any of the samples. This was not surprising, as they were not known to be produced by T cells, although IL-15 had been detected in intestinal intraepithelial lymphocytes (11). We have also examined the expression of Fas and FasL. Fas was not detected in naïve T cells 
Table 1. mRNA expression of cytokines, Fas, FasL and FLIP of naïve and activated $\gamma \delta$ T cells after TCR cross-linking

\begin{tabular}{|c|c|c|c|c|c|c|c|c|c|c|c|c|c|c|c|c|c|c|c|}
\hline \multirow[b]{2}{*}{ Time (h) } & \multicolumn{3}{|c|}{ Fas } & \multicolumn{3}{|c|}{ FasL } & \multicolumn{3}{|c|}{ FLIP } & \multicolumn{3}{|c|}{ IL-2 } & \multicolumn{3}{|c|}{ IL-4 } & \multicolumn{2}{|c|}{ TNF $\alpha$} & \multicolumn{2}{|c|}{ IFN $\gamma$} \\
\hline & $\mathbf{N}$ & $\mathbf{A}$ & CsA & $\mathbf{N}$ & $\mathbf{A}$ & CsA & $\mathbf{N}$ & $\mathbf{A}$ & CsA & $\mathbf{N}$ & $\mathbf{A}$ & CsA & $\mathbf{N}$ & $\mathbf{A}$ & CsA & $\mathbf{N}$ & $\mathbf{A}$ & $\mathbf{N}$ & $\mathbf{A}$ \\
\hline 0 & -- & ++ & ++ & ++ & ++ & ++ & +- & -- & -- & -- & -- & -- & -- & +- & ++ & ++ & -- & -- & ++ \\
\hline 4 & -- & ++ & ++ & ++ & ++ & ++ & ++ & -- & ++ & -- & ++ & -- & -- & ++ & ++ & ++ & ++ & -- & ++ \\
\hline 8 & -- & ++ & ++ & +- & ++ & ++ & -- & -- & -- & -- & -- & -- & -- & +- & -- & +- & -- & -- & ++ \\
\hline 12 & -- & ++ & ++ & +- & ++ & ++ & +- & -- & -- & -- & -- & -- & -- & -- & -- & + & -- & -- & -- \\
\hline 24 & ND & - & $\mathrm{ND}$ & - & ND & + & + & - & + & - & - & - & - & - & - & - & - & ND & - \\
\hline
\end{tabular}

mRNA expression was examined by RT-PCR for naïve (N), activated (A) and CsA treated $\gamma \delta$ T cells at various time-points after stimulation through MACS. The data set for each time-point for both naïve and activated cells represents two sets of experiments from different cultures, where + denotes the presence and-the absence of the mRNA in question. The "+/-" indicates the situation, where one experimental data set showed the presence of the given mRNA, whereas the other set showed its absence. If both experiments were positive or negative, it is denoted as ++ or -- , respectively. ND denotes not determined as several samples at t24 showed degradation of RNA and did not show a consistent signal for $\beta$-actin amplification. The rest of the data for t 24 is from one experiment.

while it was present at all time-points in activated $\mathrm{T}$ cells. In contrast, FasL was detected in both naïve and activated cells, with a stronger expression in activated T cells (Fig. 3). cFLIP was detected in naïve but not activated T cells, suggesting an anti-apoptotic protective role of cFLIP in naïve $\gamma \delta \mathrm{T}$ cells. In all our RT-PCR analyses, the negative fractions, which had no contaminating $\gamma \delta \mathrm{T}$ cells as assessed by flow cytometry, were used for comparisons. Contaminating cells in the $\gamma \delta$ positive fraction did not contribute to the message detected, as the profile of the negative fractions did not match that of the positive fractions. Furthermore, attempts to amplify the relevant message using the amount of template from the negative fraction corresponding to the number of contaminating cells in each positive fraction sample did not give a detectable signal as that in the positive fraction.

\section{Surface Expression of Fas and FasL}

Since Fas and FasL have to be expressed on the surface of cells to exert AICD, we examined their expression on naïve and activated $\gamma \delta \mathrm{T}$ cells after stimulation. It was found that naïve $\gamma \delta \mathrm{T}$ cells did not express Fas until 24 hours after stimulation while activated $\gamma \delta \mathrm{T}$ cells expressed Fas before the

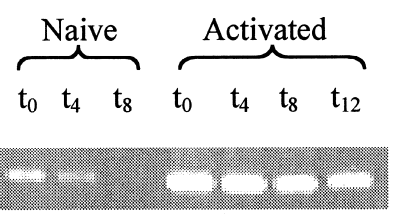

FasL

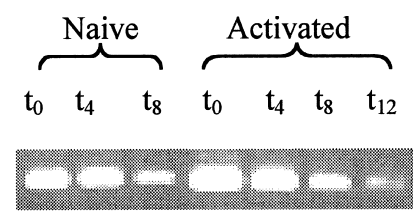

$\beta$-actin
Fig. 3. FasL and $\beta$-actin mRNA expression in naïve and activated $\gamma \delta$ T cells at various time-points after TCR cross-linking. mRNA was transcribed into cDNA before PCR amplification as described in Materials and Methods. stimulation and at all time-points examined after stimulation (Fig. 4A). Naïve cells also did not express FasL on the surface while FasL could be seen on activated $\gamma \delta \mathrm{T}$ cells most clearly at 8 hours after stimulation, although it was also present at low levels at other time-points examined with the possible exception of t0 (Fig. 4B).

\section{Cyclosporin A Inhibition of AICD}

Cyclospolrin A has been shown to inhibit AICD in other systems through the inhibition of FasL expression $(23,24)$. We found that CsA could significantly inhibit AICD of activated $\gamma \delta \mathrm{T}$ cells (Table 2 ). The treatment with CsA blocked IL-2 but not IL-4 expression. Although CsA had no effect on the Fas and FasL mRNA expression in activated $\gamma \delta \mathrm{T}$ cells, the surface expression of FasL was abrogated with CsA treatment (Fig. 4C). The expression of FLIP was detected at 4 hours for one sample, and 24 hours after stimulation for another (Table 1).

\section{Discussion}

It is known that freshly isolated peripheral $\mathrm{T}$ cells are resistant to AICD, in contrast to mature activated T cells or hybridomas, but acquire the susceptibility to AICD upon primary activation $(9,25)$. Memory T cells could also be inhibited from undergoing AICD when correctly stimulated by a foreign antigen (26), and human $\mathrm{T}$ helper $\mathrm{l}$ (Thl) lymphocytes are more susceptible to AICD than Th2 lymphocytes (27). These findings showed that regulation of AICD could depend on the cell cycle status of the cells, their cytokine environment, presence of costimulatory molecules and differential expression of apoptosis-inducers or inhibitors (28). In agreement with previous studies with $\alpha \beta$ T cells, we found that naïve human $\gamma \delta \mathrm{T}$ cells isolated from peripheral blood were resistant to AICD 
upon TCR cross-linking, in contrast to their activated counterparts. When we compared the cell cycle of naïve and activated $\gamma \delta$ T cells upon TCR cross-linking, we found that activated cells mostly remained in Gl phase throughout the 24-h examination period and exited to the sub Gl phase from Gl. The experiments were stopped at $\mathrm{t} 24$ because the majority of cells had undergone AICD by then. In contrast, naïve cells began to enter the $\mathrm{S}$ phase by 24 hours after stimulation. This is consistent with the finding that naïve $\mathrm{T}$ cells had to progress through $\mathrm{S}$ phase before acquiring sensitivity to AICD (10). The activated cells, having been in vitro culture for 11-12 days, had already undergone several divisions before the restimulation.

Activated cells also expressed IL-2 and IL-4 after stimulation whereas naïve $\mathrm{T}$ cells did not in the time

\section{A}

i)

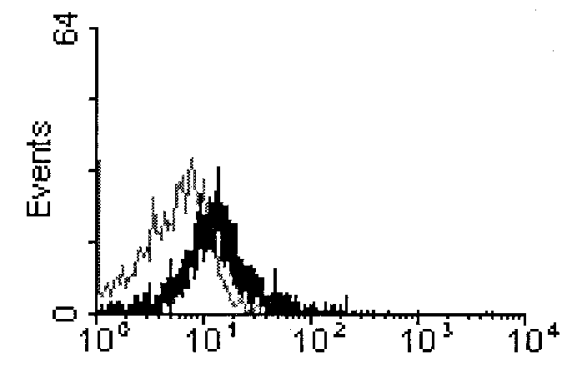

iv)

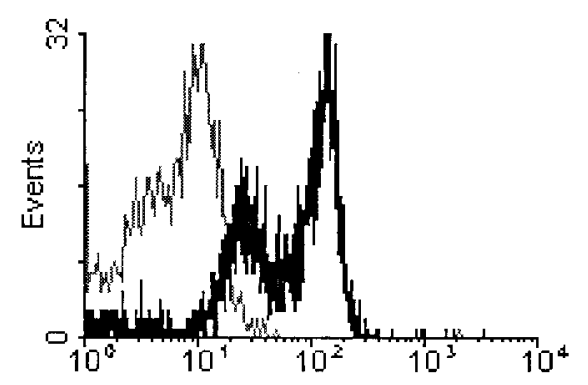

vii)

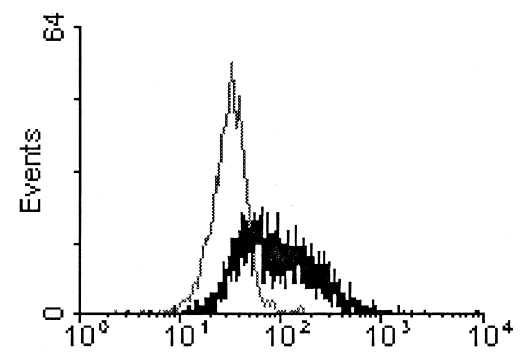

ii)

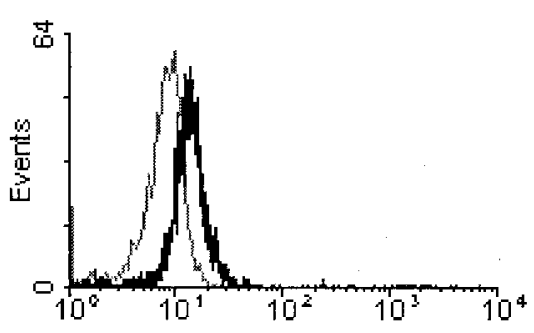

v)

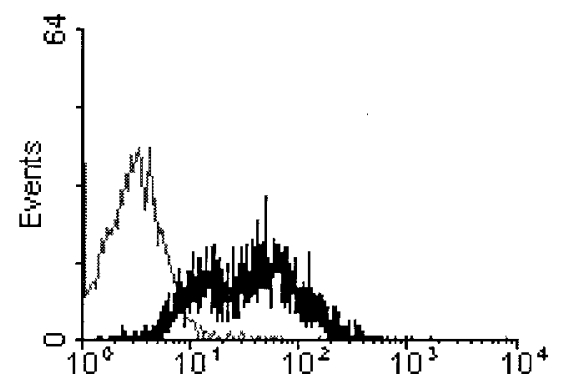

iii)

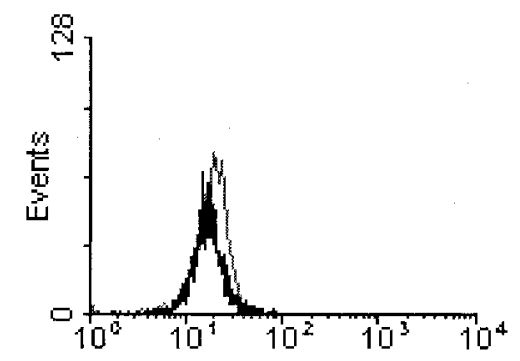

vi)

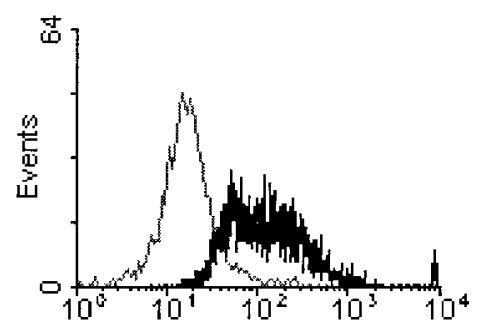

Fig. 4A. Surface expression of Fas on $\gamma \delta$ $T$ cells after stimulation. (A) Histograms in bold represent Fas staining overlaid on the negative control staining (thin lines) for naïve $\gamma \delta \mathrm{T}$ cells (i-iv) and activated $\gamma \delta$ $\mathrm{T}$ cells ( $\mathrm{v}$-viii) at different times, i.e., to (i and v), t4 (ii and vi), t8 (iii and vii) and t24 (iv and viii) after stimulation. Surface expression of FasL on $\gamma \delta$ T cells after stimulation in the absence (B) or presence (C) of CsA. Histograms in bold represent FasL staining overlaid on the negative control staining (thin lines) for naïve $\gamma \delta \mathrm{T}$ cells $(\mathbf{i}-\mathrm{v})$ and activated $\gamma \delta \mathrm{T}$ cells ( $\mathrm{vi}-\mathrm{x})$ before activation (i, vi) and at different times, i.e., to (ii and vii), t4 (iii and viii), t8 (iv and ix) and $t 24$ ( $v$ and $x)$ after stimulation, including treatment with $5 \mu \mathrm{g} / \mathrm{ml}$ of CsA (C) at t8 (i) and t24 (ii). 
B

i)

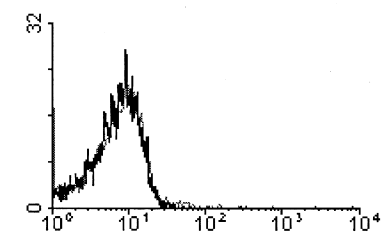

v)

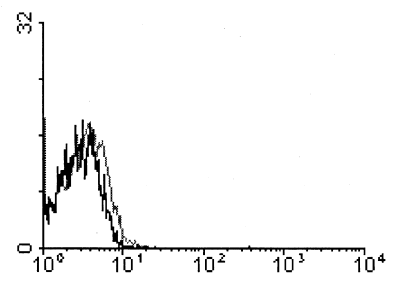

ix)

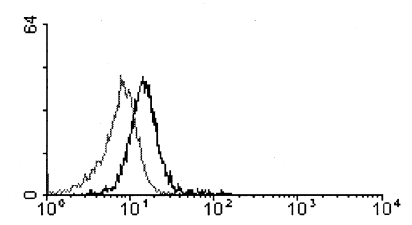

ii)

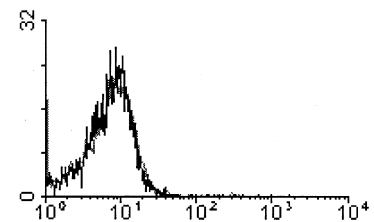

vi)

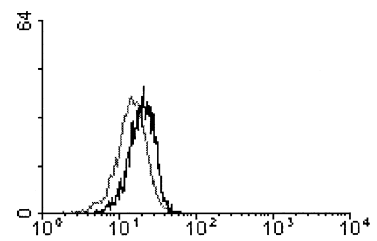

$\mathrm{x})$

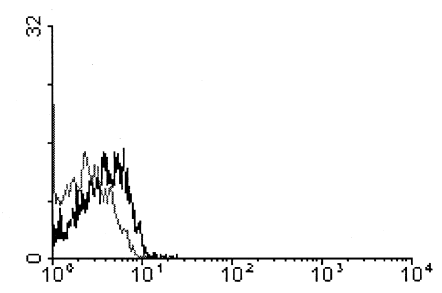

iii)

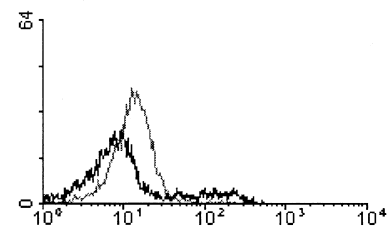

vii)

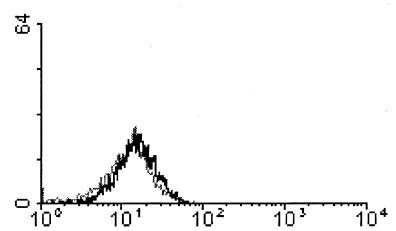

c

i)

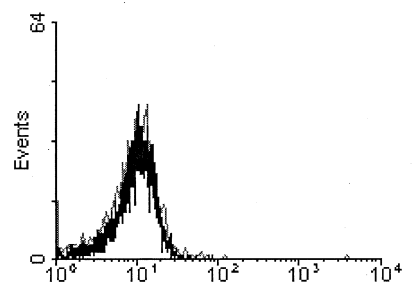

iv)

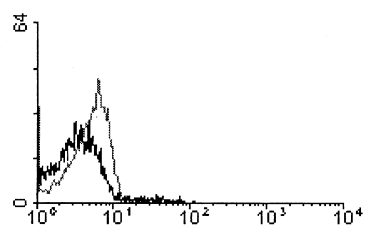

viii)

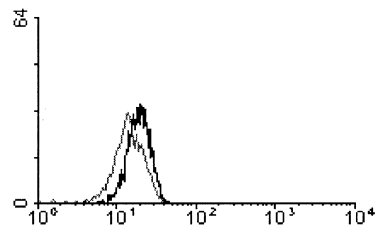

ii)

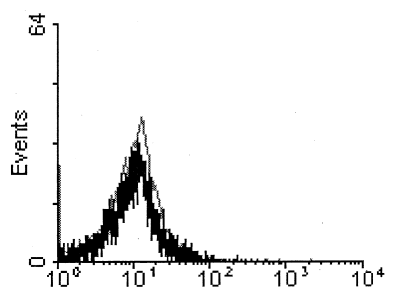

Fig. 4B and C (Continued).

period examined. As IL-2 promotes $S$ phase entry and potentiates AICD, its early expression could drive activated cells to death. This is further supported by the fact that CsA inhibited AICD and IL-2 expression in the activated cells. IL-4 expression was not inhibited by CsA, which suggests that IL-4 is not

Table 2. Effect of CsA on AICD of activated $\gamma \delta$ T cells

\begin{tabular}{lcccc}
\hline & \multicolumn{2}{c}{ Viability (\%) } & \multicolumn{2}{c}{ Apoptosis (\%) } \\
\hline Experiment 1 & T0 & T24 & T0 & T24 \\
Untreated & 96.3 & 34.2 & 3.0 & 69.6 \\
1 $\mu$ g/ml CsA & 94.2 & 60.0 & 2.5 & 17.9 \\
Experiment 2 & & & & \\
Untreated & 74.8 & 9.3 & ND & 96.1 \\
$5 \mu$ g/ml CsA & 87.7 & 57.5 & ND & 20.7 \\
& & & & \\
\hline
\end{tabular}

The percent viability was determined by trypan blue dye exclusion and percentage of apoptotic cells was determined by the annexin $\mathrm{V}$ assay as described in Materials and Methods. ND denotes not determined. The table is a composite of two separate experiments, where CsA was used at $1 \mu \mathrm{g} / \mathrm{ml}$ for one and $5 \mu \mathrm{g} / \mathrm{ml}$ for the other. involved in AICD. There was no difference in the rate and extent of cell death in naïve and activated $\mathrm{T}$ cells whether or not IL-2 was present in the culture after the TCR stimulation. This shows that it predisposes cells to AICD before the actual death signal through the TCR and not after the death signal. It is noteworthy that naïve $\gamma \delta \mathrm{T}$ cells expressed more TNF- $\alpha$ than activated T cells. Therefore, the possibility of positive contribution of TNF- $\alpha$ to this form of death appears unlikely. In addition, IFN- $\gamma$ was not detected in naïve cells, indicating that the cytokine is produced later during activation of $\gamma \delta \mathrm{T}$ cells.

Naïve T cells also did not express Fas on their surface until 24 hours after stimulation. Although they expressed FasL mRNA, the surface expression of FasL could not be detected. Thus, FasL is most likely preformed and activation leads to surface expression (29). This could account for the lack of AICD in these cells. Furthermore, cFLIP could be detected in naïve cells only, which would further prevent AICD even during later time-points when naïve cells began to express Fas. Since CsA treatment seemed to reverse the expression of cFLIP in activated cells to that of the naïve phenotype, it is possible that this could be at least partially responsible for the protection from AICD afforded by CsA. CsA has been shown to inhibit AICD in other systems by 
suppressing FasL induction $(23,24)$ through the presence of a NF-AT binding site in the promoter of FasL (30), and by preventing the down-regulation of cFLIP (29). However, another study showed that CsA inhibited cFLIP expression in memory T cells (26). In our system, we found that the expression of cFLIP in naïve $\gamma \delta \mathrm{T}$ cells correlated with survival and CsA treatment in activated cells prevented the down-regulation of cFLIP. Although FasL mRNA was still expressed in the presence of CsA, the surface expression was downregulated, suggesting that calcineurin is involved in the regulation of FasL surface expression (29). Therefore, our results support the model where cell activation leads to the upregulation of Fas, FasL and cFLIP, as in the naïve cells. At this stage, the cells are resistant to AICD. When the cells have progressed through the $S$ phase of the cell cycle and IL-2 production begins, restimulation results in AICD as CFLIP is downregulated. The regulation of cFLIP is linked to the $S$ phase progression (31), and consequently to the production of IL-2. As $\gamma \delta$ T cells have been found to be activated and expanded in numbers in various microbial infections, this mechanism of AICD in $\gamma \delta$ T cells may be crucial for maintaining $\gamma \delta \mathrm{T}$ cell homeostasis under these circumstances. The ligands recognized by $\gamma \delta \mathrm{T}$ cells are common in both microbes and man and are likely to be released when cells die during inflammation $(15,16)$. Consequently, it may be important to downregulate the $\gamma \delta$ T-cell response when the offending pathogen has been removed since chronic activation of the cells may result in autoimmunity. Understanding the mechanism of AICD also creates opportunities for possible therapeutic interventions in some retroviral and mycobacterial infections $(12,14,32-35)$, where preventing the premature deletion or increased susceptibility of $\gamma \delta$ $\mathrm{T}$ cells to apoptosis may be desirable.

\section{Acknowledgments}

We are grateful to Soh Chan Lim for technical assistance. This work was funded by grants RP3972389 and R-183-000-018-112 from the National University of Singapore and the NIH grant AI48401-02.

\section{References}

1. Kabelitz D, Pohl T, Pechhold K. (1993) Activation-induced cell death (apoptosis) of mature peripheral $\mathrm{T}$ lymphocytes. Immunol. Today 14(7): 338-339.

2. Van Parijs L, Abbas AK. (1998) Homeostasis and self-tolerance in the immune system: turning lymphocytes off. Science $\mathbf{2 8 0}$ (5361): 243-248.

3. Ju ST, Panka DJ, Cui H, Ettinger R, El-Khatib M, Sherr DH, Stanger BZ, Marshak-Rothstein A. (1995) Fas (CD95)/FasL interactions required for programmed cell death after $\mathrm{T}$ cell activation. Nature 373(6513): 444-448.

4. Dhein J, Walcak H, Baumler C, Debatin KM, Krammer PH. (1995) Autocrine T-cell suicide mediated by APO- 1/(Fas/CD95). Nature 373(6513): 438-441.

5. Brunner T, Mogil RJ, Laface D, Yoo NJ, et al. (1995) Cellautonomous Fas(CD95)/Fas-ligand interaction mediates activation-induced apoptosis in T-cell hybridomas. Nature 373(6513): 441-444.
6. Refaeli Y, Van Parijs V, London CA, Tschopp J, Abbas AK. (1998) Biochemical mechanisms of IL-2-regulated Fasmediated T cell apoptosis. Immunity 8(4): 615-623.

7. Hitoshi Y, Lorens J, Kitada S-I, Fisher J, Labarge M, Ring HZ, Francke U, Reed JC, Kinoshita S, Nolan GP. (1998) Toso, a cell surface, specific regulator of Fas-induced apoptosis in T cells. Immunity 8(4): 461-471.

8. Irmler M, Thome M, HahneA M, et al. (1997) Inhibition of death receptor signals by cellular FLIP. Nature 388(6638): 190-195.

9. Lenardo MJ. (1991) Interleukin-2 programs mouse $\alpha \beta$ T lymphocytes for apoptosis. Nature 353(6347): 858-861.

10. Radvanyi LG, Shi Y, Mills GB, Miller RG. (1996) Cell cycle progression out of Gl sensitizes primary-cultured nontransformed T cells to TCR-mediated apoptosis. Cell. Immunol. 170(2): 260-273.

11. Chu C-L, Chen S-S, Wu T-S, Kuo S-C, Liao N-S. (1999) Differential effects of IL-2 and IL-15 on the death and survival of activated TCR $\gamma \delta+$ intestinal intraepithelial lymphocytes. J. Immunol. 162(4): 1896-1903.

12. Li B, Bassiri H, Rossman MD, et al. (1998) Involvement of the Fas/FasLigand pathway in activation-induced cell death of mycobacteria-reactive human $\gamma \delta$ T cells: a mechanism for the loss of $\gamma \delta \mathrm{T}$ cells in patients with pulmonary tuberculosis. J. Immunol. 161(4): 1558-1567.

13. Poccia F, Gougeon M-L, Bonneville M, et al. (1998) Innate T-cell immunity to nonpeptidic antigens. Immunol. Today 19(6): 253256.

14. Poccia F, Agrati C, Ippolito G, Colizzi V, Malkovsky M. (2001) Natural T cell immunity to intracellular pathogens and nonpeptidic immunoregulatory drugs. Curr. Mol. Med. 1: 137-151.

15. Constant P, Davodeau F, Peyrat M-A, et al. (1994) Stimulation of $\gamma \delta \mathrm{T}$ cells by nonpeptidic mycobacterial ligands. Science 264(5156): 267-270.

16. Tanaka Y, Morita CT, Tanaka Y, Nieves E, Brenner MB, Bloom BR. (1995) Natural and synthetic non-peptide antigens recognized by human $\gamma \delta$ T cells. Nature 375(6527): 155-158.

17. Evans PS, Enders PJ, Yin C, Ruckwardt T, Malkovsky M, Pauza CD. (2001) In vitro stimulation with a non-peptidic alkylphosphate antigen expands cells expressing the $\mathrm{V} \gamma 2$ $\mathrm{J} \gamma 1.2 / \mathrm{V} \delta 2 \mathrm{~T}$ cell receptors. Immunology, in press.

18. Bukowski JF, Morita CT, Brenner MB. (1999) Human $\gamma \delta$ $\mathrm{T}$ cells recognize alkylamines derived from microbes, edible plants, and tea: implications for innate immunity. Immunity 11(1): 57-65.

19. Wang P, Malkovsky M. (2000) Different roles of the CD2 and LFA-1 T cell co-receptors in regulating cytotoxic, proliferative and cytokine responses of $\mathrm{V} \gamma 9 / \mathrm{V} \delta 2$ T cells. Mol. Med. 6: 196-207.

20. Gan YH, Malkovsky M. (1996) Mechanisms of simian $\gamma \delta$ $\mathrm{T}$ cell cytotoxicity against tumor and immunodeficiency virus-infected cells. Immunol. Lett. 49(3): 191-196.

21. Gan YH, Wallace M, Malkovsky M. (2001) Fas-dependent, activation-induced cell death of $\gamma \delta$ T cells. J. Biol. Regul. Homeost. Agents, in press.

22. Salerno A, Francesco D. (1998) Role of $\gamma \delta$ T lymphocytes in immune response in humans and mice. Crit. Rev. Immunol. 18(4): 327-357.

23. Fournel S, Genestier L, Robinet E, Flacher M, Revillard J-P. Human T cells require IL-2 but not Gl/S transition to acquire susceptibility to Fas-mediated apoptosis. (1996) J. Immunol. 157(10): 4309-4315.

24. Brunner T, Yoo NJ, Laface D, Ware CF, Green DR. (1996) Activation-induced cell death in murine $\mathrm{T}$ cell hybridomas. Differential regulation of Fas(CD95) versus Fas ligand expression by cyclosporin $\mathrm{A}$ and FK506. Int. Immunol. 8(7): $1017-1026$.

25. Russel JH, White CL, Loh DY, Meleedey-Rey P. (1991) Receptor-stimulated death pathway is opened by antigen in mature T cells. Proc. Natl. Acad. Sci. USA 88(6): 2151-2155.

26. Di Somma MM, Somma F, Montani MSG, Mangiacasale R, Cundari E, Piccolella E. (1999) TCR engagement regulates 
differential responsiveness of human memory $\mathrm{T}$ cells to Fas (CD95)-mediated apoptosis. J. Immunol. 162(7): 38513858.

27. Varadhachary AR, Perdow SN, Hu C, Ramanarayanan M, Salgame P. (1997) Differential ability of T cell subsets to undergo activation-induced cell death. Proc. Natl. Acad. Sci. USA. 94(11): $5778-5783$.

28. Janssen O, Sanzenbacher R, Kabelitz D. (2000) Regulation of activation-induced cell death of mature T-lymphocyte populations. Cell Tissue Res. 301(1): 85-99.

29. Toth R, Szegezdi E, Molnar G, Lord JM, Fesus L, Szondy Z. (1999) Regulation of cell surface expression of Fas (CD95) ligand and susceptibility to Fas-mediated apoptosis in activation-induced $T$ cell death involves calcineurin and protein kinase C, respectively. Eur. J. Immunol. 29(2): 383-393.

30. Latinis KM, Norian LA, Eliason SL, Koretsky GA. (1997) Two NFAT transcription factor binding sites participate in the regulation of CD95 (Fas) ligand expression in activated human T cells. J. Biol. Chem. 272(50): 31427-31434.
31. Algeciras-Schimnich A, Griffith TS, Lynch DH, Paya CV. (1999) Cell cycle-dependent regulation of FLIP levels and susceptibility to Fas-mediated apoptosis. J. Immunol. 162(9): 5205-5211.

32. Poccia F, Boullier S, Lecoeur $\mathrm{H}$, et al. (1996) Peripheral $\mathrm{V} \gamma 9 / \mathrm{V} \delta 2 \mathrm{~T}$ cell deletion and anergy to nonpeptidic mycobacterial antigens in asymptomatic HIV-1-infected persons. J. Immunol. 157(1): 449-461.

33. Wallace M, Scharko AM, Pauza CD, et al. (1997) Functional $\gamma \delta$ T-lymphocyte defect associated with human immunodeficiency virus infections. Mol. Med. 3(1): 60-71.

34. Malkovsky M, Wallace M, Fournié J-J, Fisch P, Poccia F, Gougeon, M-L. (2000) Alternative cytotoxic effector mechanisms in infections with immunodeficiency viruses: $\gamma \delta \mathrm{T}$ lymphocytes and NK cells. AIDS 14: S175-S186.

35. Poccia F, Malkovsky M, Pollak A, et al. (1999) In vivo $\gamma \delta$ T-cell priming to mycobacterial antigens by primary $M$. tuberculosis infection and exposure to nonpeptidic ligands. Mol. Med. 5(7): 471-476. 